University of Nebraska - Lincoln

DigitalCommons@University of Nebraska - Lincoln

Robert Katz Publications

Research Papers in Physics and Astronomy

August 1976

\title{
Supralinearity of Thermoluminescent Dosimeters
}

Leif Larsson

University of Nebraska-Lincoln

Robert Katz

University of Nebraska-Lincoln, rkatz2@unl.edu

Follow this and additional works at: https://digitalcommons.unl.edu/physicskatz

Part of the Physics Commons

Larsson, Leif and Katz, Robert, "Supralinearity of Thermoluminescent Dosimeters" (1976). Robert Katz Publications. 97.

https://digitalcommons.unl.edu/physicskatz/97

This Article is brought to you for free and open access by the Research Papers in Physics and Astronomy at DigitalCommons@University of Nebraska - Lincoln. It has been accepted for inclusion in Robert Katz Publications by an authorized administrator of DigitalCommons@University of Nebraska - Lincoln. 
Published in Nuclear Instruments and Methods 138 (1976), pp. 631-636.

Copyright (C) North-Holland Publishing Co. /Elsevier. Used by permission.

http://www.sciencedirect.com/science/journal/01675087

Supported by the United States Energy Research and Development Administration, and the National Science Foundation (RANN).

Submitted August 16, 1976.

\title{
Supralinearity of Thermoluminescent Dosimeters
}

\author{
Leif Larsson and Robert Katz * \\ University of Nebraska-Lincoln, Lincoln, Nebraska 68588, U.S.A. \\ * Corresponding author.
}

\begin{abstract}
The supralinear response of thermoluminescent dosimeters (TLDs) after irradiation with gamma- or beta-rays can be decomposed by use of the cumulative Poisson distribution into a sum of 1-or-more and 2-or-more hit components, suggesting the coexistence of both types of trap structures. Following the response of other 1-hit detectors, and of biological cells to energetic heavy ions, we expect and find that the supralinear response tends to disappear with alpha particle, and with neutron irradiation, and that the supralinear, or 2-hit, component tends to be more responsive to high LET (Linear Energy Transfer) radiations. Since the response of biological cells to gamma-rays is also supralinear, the supralinear component of the TLD response can be expected to have the capacity to mimic the response of biological cells and tissues to radiations of different quality.
\end{abstract}

\section{Introduction}

The response of a large number of detectors to ionizing radiations of different quality is characterized by the theory of track structure, ${ }^{1-8}$ according to whether the detectors can be described as having 1-or-more hit, c-or-more hit (in a single target) response, or as requiring 1-or-more hits in each of $m$ targets in a sensitive volume. Included among 1-hit detectors are many solid state systems (most photographic emulsions, scintillators, color center formation, radical formation in organic molecules) and some biological systems (the inactivation of enzymes and viruses or the creation of single strand breaks in DNA). Certain underdeveloped photographic emulsions have been identified as c-or-more hit detectors, with hittedness ranging to 8 . The $m$-target detector model has been applied to many biological cells and tissues.

If 1-hit response to electron beams is interpreted to imply that the observed end-point can be achieved after the passage of a single electron through the sensitive target, then biological cells require the passage of more than 1 electron through the cell nucleus, through $m$ or more targets, to inactivate the cell. A c-or-more hit detector requires the passage of $c$-or-more electrons through the sensitive target to produce the observed end-point. Since delta-rays (secondary electrons) are clustered around the path of an energetic heavy ion, the passage of a single ion through the nucleus of a cell, or through the sensitive target of a $c$-hit detector, may result in its (in)activation. Thus while the response of such a detector to beta-rays, or to the secondary electrons from gamma-rays, is supralinear, its response to heavy ions is linear, or more accurately, exponentially saturating.
In radiobiology, one speaks of the Relative Biological Effectiveness (RBE) of two different radiation fields, of different LET (Linear Energy Transfer, or stopping power) as the ratio of the doses to produce the same effect. The reference radiation is often taken to be gammarays, thus defined to have $R B E=1$. Typically we find that the RBE is a function of LET, and since the doseeffect relationships may have different shapes, the RBE may also be a function of the dose, or of the effect level. The terminology has carried over to other detectors that are not biological.

The concentration of delta-rays around the path of a high LET particle leads to energy wastage, or overkill, with 1-hit detectors, so that the RBE of a 1-hit detector is never greater than 1 , and typically declines with an increase in LET. This concentration of delta-rays leads to an enhanced response in $m$-target or $c$-hit detectors, at low dose levels, so that these detectors (say, biological cells) display an RBE which first increases with an increase in LET, passing through a maximum, and then declining when more than half the sensitive targets intersected by the ion are (in)activated, so that a further increase in LET results in energy wastage in the targets already killed. The question as to what does one mean by low LET or by high LET - compared to what-must be answered by track theory.

\section{Track theory}

The structure of the track of an energetic heavy ion is built from the radial distribution of "local dose" deposited by delta-rays (secondary electrons) in concentric cylindrical shells about the ion's path. Typically we 
calculate the average (over many identical ions) radial distribution in local dose by a series of constructs and approximations, involving a delta-ray distribution formula, an assumption of the initial angular distribution of the ejected electrons, electron range-energy relations, or experimentally based electron energy dissipation algorithms, and so on. From such calculations we find a dominant result that the radial distribution in local dose varies as $z^{2} / \beta^{2} t^{2}$, where $z$ is the effective charge number of the ion (accomodating for charge pick-up), $\beta$ is its relative speed, and $t$ is the radial distance from the ion's path. The local dose drops to zero as we approach and exceed distances $t=\tau$ corresponding to the outermost radial reach of the most energetic delta-rays permitted by collision kinematics. The radial distribution of local dose $\bar{E}(t)$, deposited in a sensitive cylinder of radius $a_{0}$ whose axis is parallel to and at radial distance $t$ from the

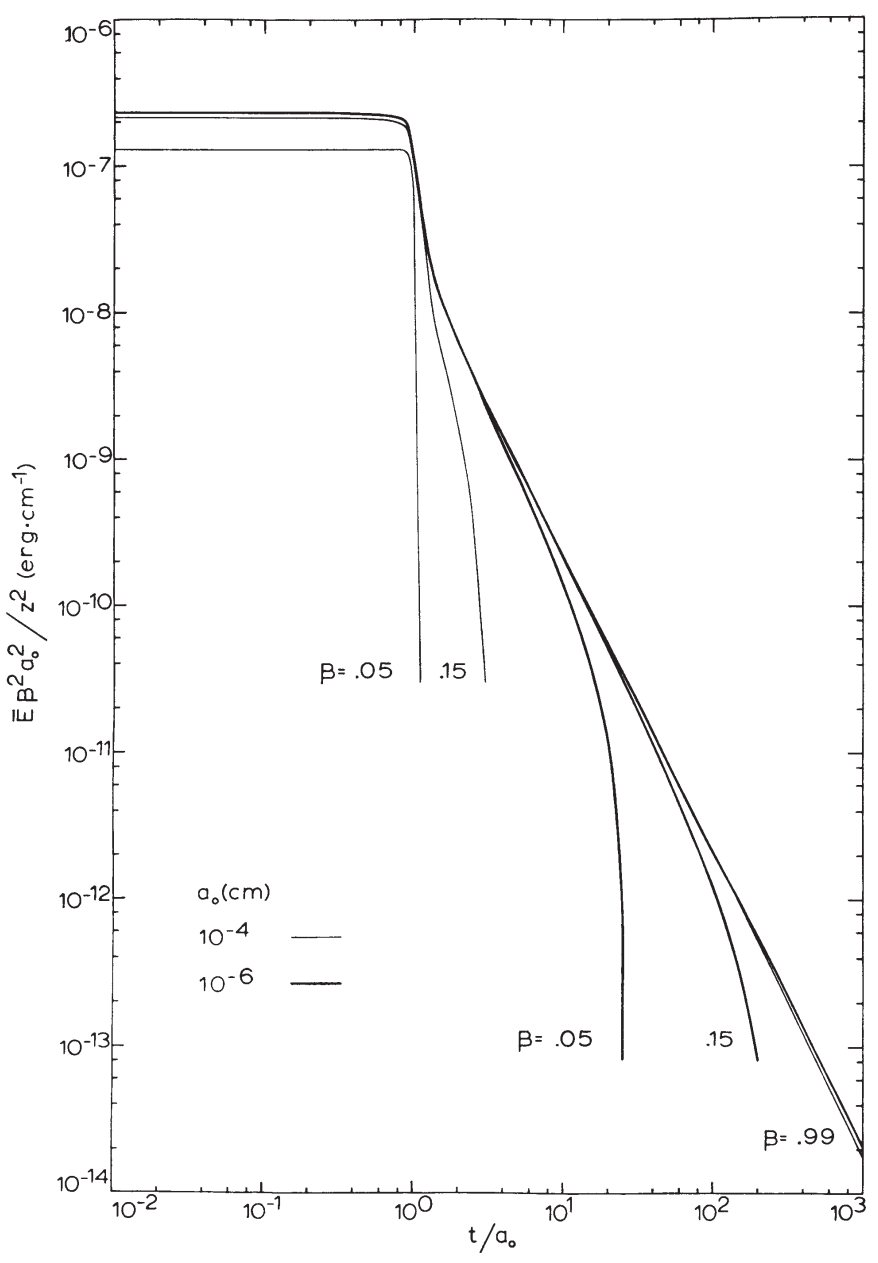

Figure 1. Radial distribution of local dose, $\bar{E}(t)$, deposited in a near circular cylinder of radius $a_{0}$ whose axis is parallel to and at radial distance $t$ from the path of an ion of effective charge number $z$ moving at relative speed $\beta$ through water. ion's path, is shown in Figure 1, for a particle passing through water.

We consider detectors to be characterized by a sensitive target, of radius $a_{0}$, and by a characteristic dose $E_{0}$ of gamma-rays. We take the ratio $\bar{E} / E_{0}$ (for the local dose from delta-rays, or $D / E_{0}$ for the macroscopic dose from gamma-rays) $=A$, to represent the average number of "hits" per target. Thus $E_{0}$ is the macroscopic dose of gamma-rays at which there is an average of 1 hit per target. By the word "hit" we mean an interaction leading to a scored event. Thus $E_{0}$ and $a_{0}$ are two of our detector parameters, which join the number $m$ or $c$. We cannot parameterize a detector with fewer than three parameters unless there are internal relations joining these numbers based on the fundamental nature of the detector.

In many cases the character of the response of the detector to heavy particles is associated with the response of sensitive elements through which the particle passes, when $t / a_{0}<1$. From Figure 1 we note that for small radial distances, and sufficiently high particle speeds that the distance $\tau$ is not a limiting feature, we find for $t<a_{0}$ :

$$
\bar{E} \beta^{2} a_{0}^{2} / z^{2}=2 \times 10^{-7} \mathrm{erg} / \mathrm{cm},
$$

where the numerical value on the right-hand side of the equation is calculated for water, and changes for different media, though the form of the figure remains essentially the same. Thus we find for $t<a_{0}$ :

$$
\bar{E} / E_{0}=z^{2} / \beta^{2}\left(E_{0} a_{0}^{2} / 2 \times 10^{-7} \mathrm{erg} / \mathrm{cm}\right) .
$$

It is convenient to define

$$
\kappa=E_{0} a_{0}^{2} / 2 \times 10^{-7} \mathrm{erg} / \mathrm{cm},
$$

and to use the quantity $\kappa$ as a universal detector parameter through which we can characterize low and high LET radiation effects. We find that it is convenient to characterize

$$
\begin{aligned}
& \text { low LET radiations: } z^{2} / \kappa \beta^{2}<0.1, \\
& \text { high LET radiations: } z^{2} / \kappa \beta^{2} \geq 0.1,
\end{aligned}
$$

so that what is low LET or what is high LET depends on the detector perceiving the radiation. We have found values of $\kappa$ ranging from 10 to $10^{4}$ in different detectors. For a number of biological cells $\kappa$ is in the neighborhood of 1,000 , making it appear that the boundary between low and high LET is clearer than in fact it is, when expressed in units of stopping power.

To represent detector response we have used two functional forms, called the multi-target and the multihit ${ }^{9}$ models, in which we take the probability that the 
sensitive element of a detector (the photographic grain, the nucleus of a biological cell, the activation center in a scintillator) will be (in)activated in a uniform field of gamma-rays at dose $D$ to be multi-target:

$$
P(m, A)=\left(1-\mathrm{e}^{-A}\right)^{m}, \quad \text { or }
$$

multi-hit:

$$
P(c, A)=\sum_{x=c}^{\infty} \frac{A^{x} \mathrm{e}^{-A}}{x !}=1-\sum_{x=0}^{c-1} \frac{A^{x} \mathrm{e}^{-A}}{x !},
$$

where:

$$
A=D / E_{0} .
$$

In a 1-or-more hit detector, with a single target, $c=m$ $=1$, and the expressions are identical. Their graphs are of very similar form (though at different values of $A$ ) for low $m$ or $c$, and differ increasingly as $m$ or $c$ are larger than 3. For the special cases of 1- and 2-hit detectors of interest here, the expression equation (6) reduces to

$$
\begin{aligned}
& \text { 1-or-more hit: } P(1, A)=1-\mathrm{e}^{-A}, \\
& \text { 2-or-more hits; } P(2, A)=1-(1+A) \mathrm{e}^{-A} \text {. }
\end{aligned}
$$

When we know the quantities $E_{0}, a$, and $m$ or $c$, for a detector, we can find the radial distribution of (in)activation probability about an ion's path, by applying equation (5) or (6) to Figure 1. Thus we can generate a computer simulation of the track of a heavy ion in nuclear emulsion, ${ }^{7}$ or we can integrate radially to find the cross-section for the inactivation of an enzyme molecule, ${ }^{1}$ or the relative pulse height in a scintillation counter, $^{2-5}$ or the surviving fraction of a collection of irradiated biological cells. ${ }^{4}$

To describe the effects of heavy ions on $m$-target detectors (biological cells) or on c-hit detectors we must introduce the concepts of "ion-kill" and "gamma-kill."

By ion-kill we mean that the sensitive element of a detector is (in)activated by a single heavy ion, when the fluctuating delta-ray density is sufficient to produce the requisite number of hits.

By gamma-kill we mean that the number of delta-rays from an ion is insufficient for inactivation, but that sublethal damage is done by one ion, and that the contribution from the delta-rays of a second or a third ion add together to produce the (in)activation. This effect is parallel to the accumulation of sub-lethal damage from the secondary electrons ejected from the medium by the absorption of gamma-rays.

When the gamma-kill mode is dominant, we interpret the interaction as from a many-hit or a many-target process, for delta-rays from several ions must hit the target to (in)activate it. In radiobiology we see a survival curve with a shoulder.
When the ion-kill mode is dominant, we interpret the interaction as a 1-hit process, for a target need be hit by only one ion to (in)activate it. In radiobiology we see exponential survival curves, without shoulders.

We begin to see the effects of ion-kill as $z^{2} / \kappa \beta^{2}$ exceeds 0.1. It is for this reason that we have used this value in equation (4) to discriminate between low and high LET radiation.

If we think of the irradiation with a beam of heavy ions as a two-step process, in which the survivors of the ion-kill component of the irradiation are the initial population to be exposed to gamma-kill, we can see that the response to a beam of ions passes from many-hit or many-target to one-hit as we pass from low to high LET radiation.

Although these considerations were first developed from the application of track theory to radiobiology, they clearly must apply to any detector exhibiting a supralinear response to gamma-rays.

For our discussion of thermoluminescent dosimeters it is convenient to display the incremental response of a 1- and a 2-hit detector $\mathrm{d} P / \mathrm{d} A$, as a function of the "relative dose," $A$. In Figure 2, this is plotted as the relative efficiency, normalized to $100 \%$, at maximum. We also show $P(c, A)$ vs $A$ in Figure 3 .

If a detector contains both linear (1-hit) and supralinear (2-hit) elements, the value of $E_{0}$ need not be the same for the different classes of sensitive elements, nor can we expect that the number of sensitive elements in the two classes is the same. Thus a real detector containing both classes of elements may exhibit their contributions shifted horizontally, when plotted as a function of dose, and normalized to different vertical values.

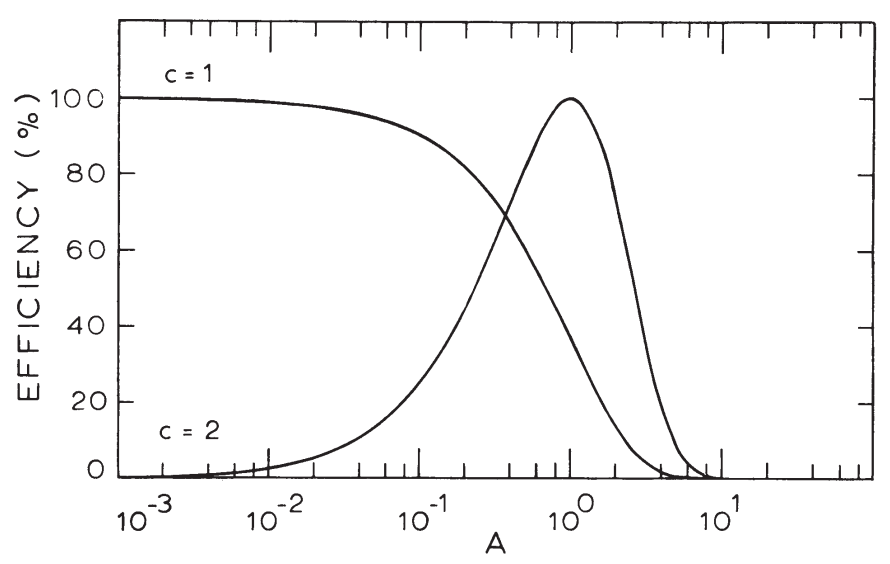

Figure 2. "Efficiency" of 1-hit and 2-hit detectors plotted as a function of the relative dose, $A$. 


\section{Thermoluminescent dosimeters}

The response of thermoluminescent dosimeters (TLDs) to ionizing radiations is described in books by Becker, ${ }^{10}$ by Cameron et al. ${ }^{11}$ in papers published in four international conferences held in 1965, ${ }^{12} 1968,13$ 1971, ${ }^{14}$ and $1974,{ }^{15}$ as well as in the periodical literature.

After irradiation with low LET radiations, like gamma- or beta-rays, TLDs typically exhibit a linear response at low dose levels, and a supralinear response at higher doses. The supralinear component may make its appearance at exposures from $1 \mathrm{R}$ to $1000 \mathrm{R}$, depending on the crystal, its doping, its annealing, and previous irradiation history. A saturation in response is often observed at exposures in the neighborhood of $10^{5}-10^{6} \mathrm{R}$. In some crystals the supralinear response disappears after annealing.

The emitted light is measured as a function of time or temperature, as the crystals are heated in an oven according to a prescribed cycle. The light is typically emitted in peaks at different temperatures, presumably as electrons are released from different trap structures. The light yield is sometimes represented as the peak height of a fixed peak in the glow curve, or as the maximum height of the glow curve, or as the total integrated light between some fixed temperature limits. The response may be linear in one peak and supralinear in another. The supralinear response is often associated with the higher temperature peaks.

In Figures 4-6 we display the response of three phosphors, TLD-200 $\left(\mathrm{CaF}_{2}: \mathrm{Dy}\right)$ for peaks $5+6,{ }^{16} \mathrm{BeO}$ for the glow peak maximum, ${ }^{17}$ and $\mathrm{H} 63 \mathrm{~A}(\mathrm{LiF})$ for the $110^{\circ}$ peak, ${ }^{18}$ as decomposed into 1-or-more and 2-or-more hit responses, by visual fitting techniques. Values of $E_{0}$ and of the relative contributions of the 1-hit and the 2hit traps are shown in table 1 , for the particular readout made of these responses.

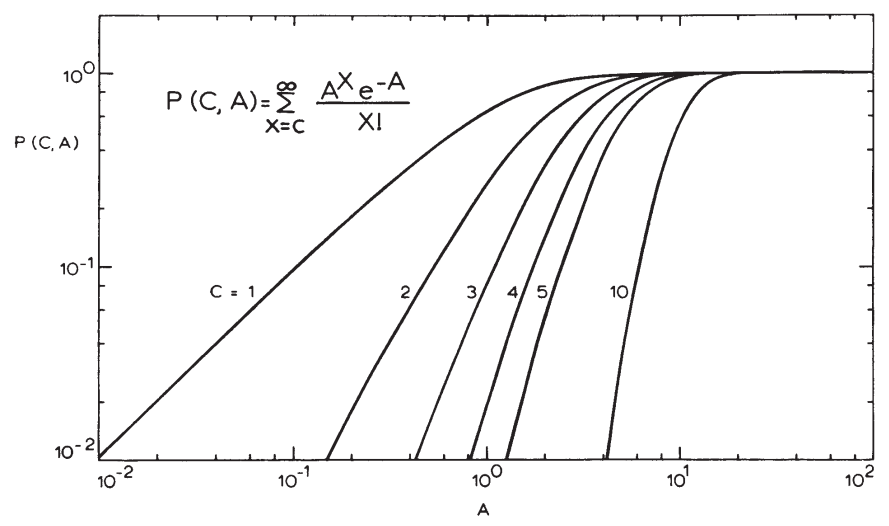

Figure 3. The cumulative Poisson distribution $P(c, A)$ vs the number of trials $A$, representing the probability that $c$-or-more hits are scored when there is an average of $A$ trials per target.
The transition from supralinearity with low LET radiations (gamma- and beta-rays, fast protons) to linearity with high LET radiations (slow He ions, stopping alphaparticles, neutron interactions, heavy ions) has been observed by a number of investigators. ${ }^{17,} 19-24$

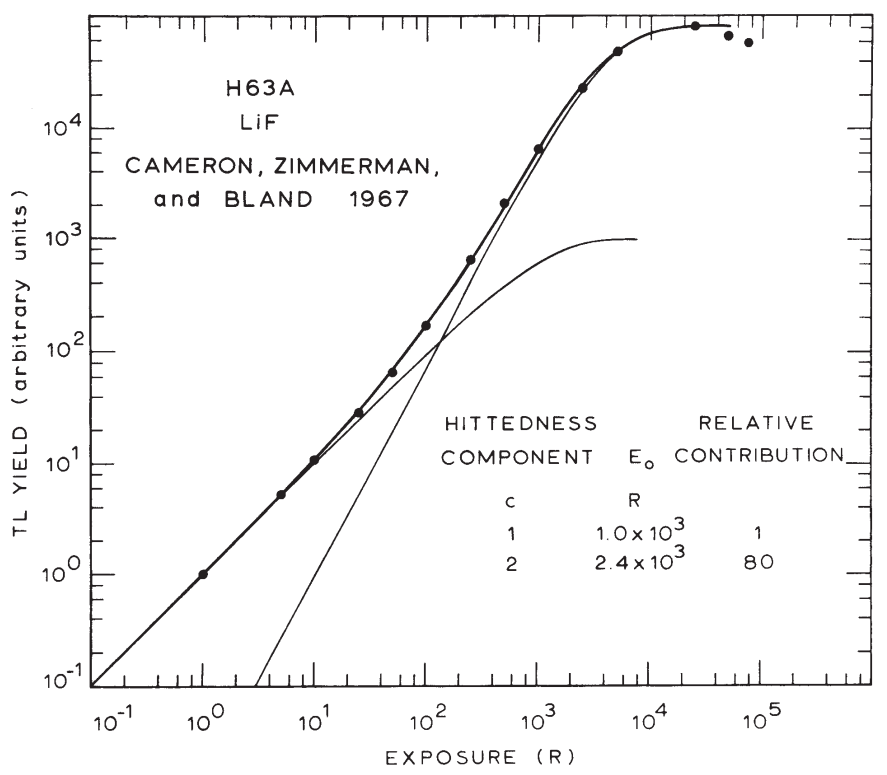

Figure 4. The heavy line shown superimposed over experimental data ${ }^{18}$ for a $\mathrm{LiF}$ dosimeter is the sum of the response represented by two light lines, for 1-hit and 2-hit traps.

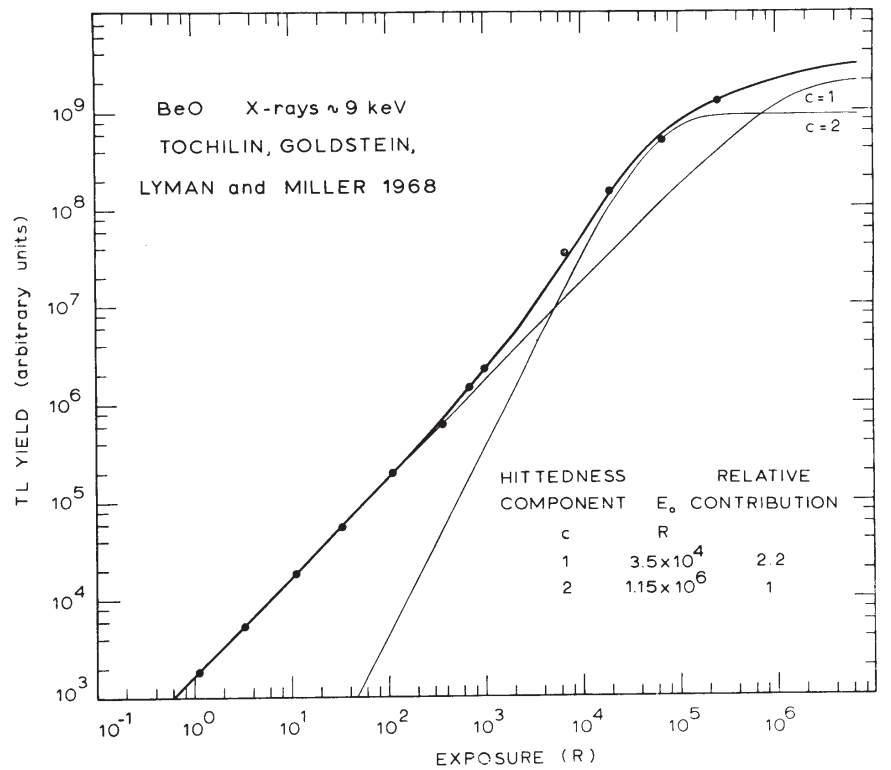

Figure 5. BeO. ${ }^{17}$ See caption to Figure 4. 
Table 1. Properties of some thermoluminescent dosimeters.

\begin{tabular}{|c|c|c|c|c|c|}
\hline \multirow[t]{2}{*}{ Substance } & \multirow[t]{2}{*}{ Reference } & \multicolumn{2}{|c|}{ 1-hit trap } & \multicolumn{2}{|c|}{ 2-hit trap } \\
\hline & & $\begin{array}{l}E_{0} \\
(\mathrm{R})\end{array}$ & $\begin{array}{c}\text { Relative } \\
\text { contribution }\end{array}$ & $\begin{array}{l}E_{0} \\
(\mathrm{R})\end{array}$ & $\begin{array}{l}\text { Relative } \\
\text { contribution }\end{array}$ \\
\hline H63A (LiF) & Cameron et al. ${ }^{18}$ & $1.0 \times 10^{3}$ & 1 & $2.4 \times 10^{3}$ & 80 \\
\hline $\mathrm{BeO}$ & Tochilin et al. ${ }^{17}$ & $3.5 \times 10^{4}$ & 2.2 & $1.2 \times 10^{6}$ & 1 \\
\hline
\end{tabular}

The situation in regard to the variation of RBE with LET is not so clear. Some have noted a decline in response with an increase in LET, 19, 21, 22, 25 as would be appropriate to a 1-hit detector. At the same time there are indications ${ }^{19}$ that the RBE of thermal neutrons exceeds 1 , in a high temperature peak of TLD-100, and this has provided the basis of a system for the separate identification of neutron and gamma-exposures, ${ }^{26}$ by considering the ratio of the signals obtained from an initial reading of the light released between 150 and $250{ }^{\circ} \mathrm{C}$, to that from a second reading at $325^{\circ} \mathrm{C}$. The second reading is $1.5 \%$ of the first if the dosimeter has been exposed only to gamma-rays (below $100 \mathrm{R}$ ) and is 17\% if exposed only to neutrons. In the light of track theory these observations depend on whether one is reading 1-hit or 2-hit traps, and on the balance between ion-kill and gammakill in the neutron irradiations.

While their data do not lend themselves to ready interpretation in terms of the present model, Tochilin et al. ${ }^{17}$ have noted a possible connection between a high

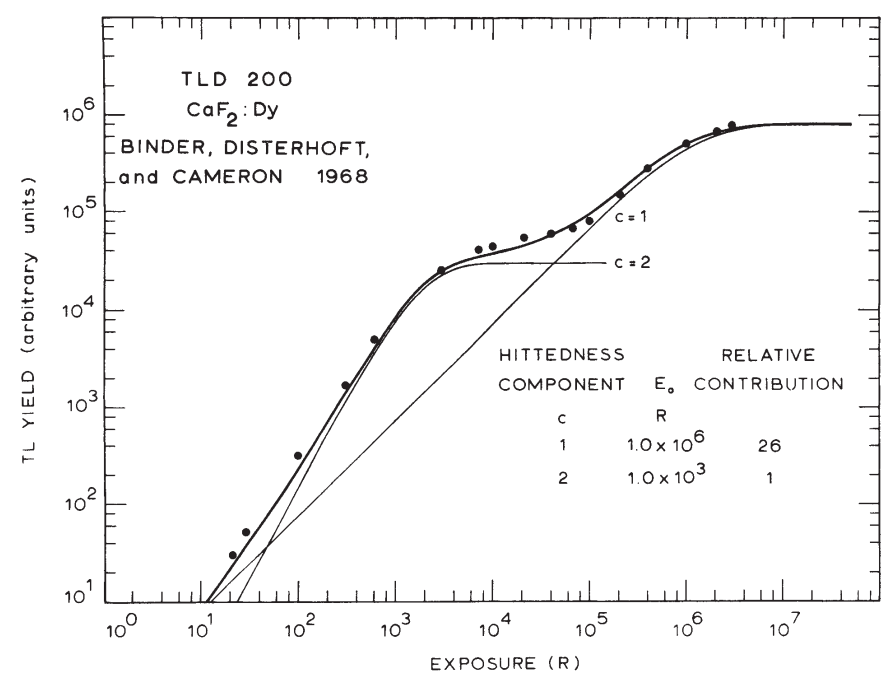

Figure 6. $\mathrm{CaF}_{2}$ : Dy. ${ }^{16}$ See caption to Figure 4. degree of supralinearity with low LET radiations, and an increasing TL response per Rad with high LET radiations, as a result of their comparisons of the low and high LET responses of $\mathrm{BeO}, \mathrm{Li}_{2} \mathrm{~B}_{4} \mathrm{O}_{7}: \mathrm{Mn}$, and $\mathrm{LiF}$. For $\mathrm{BeO}$, an "RBE" relative to ${ }^{60} \mathrm{Co}$ gamma-rays of about 2 was observed, for heavy ions.

A much clearer connection between RBE to high LET radiations and supralinearity is made by Jaek et al., ${ }^{23}$ who claim values of $200-400$ for special peaks in CaS phosphors irradiated with alpha particles, and suggest that these substances may therefore be used for the selective registration of neutrons above a gamma background.

The numerical values of the RBE depend on the dose level, with the highest values to be obtained at the lowest doses, ${ }^{8}$ if the present model is applicable to these experiments. This comes about simply enough, from the comparison of doses at which the effects are the same, in two dose-response curves having different shapes, as in Figure 3.

We must point out that the analysis of Jaek et al. ${ }^{23}$ is very similar in perspective to our own, in connecting the response of the dosimeter to gamma-rays to its response to high LET particles through the local dose in the neighborhood of the particle track, although that analysis is a qualitative one.

We propose no model of a trap structure which might account for 2-or-more hittedness.

The preceding paragraphs give some substance to the possible existence of 1-hit and 2-hit traps, and offer a conceptual structure in terms of which we can account for the presently observed response of TLDs to high LET radiations.

Data are not available from which a more precise test of the model can be made.

The response of the 1-hit traps to high LET radiations is in good accord with the theory. ${ }^{21}$

It is the 2-hit trap structure which needs further elaboration. 


\section{References}

1. J. J. Butts and R. Katz, Radiat. Res. 30 (1967) 855.

2. R. Katz and E. J. Kobetich, Phys. Rev. 170 (1968) 397.

3. R. Katz and E. J. Kobetich, Phys. Rev. 186 (1969) 344.

4. R. Katz, B. Ackerson, M. Homayoonfar, and S. C. Sharma, Radiat. Res. 47 (1971) 402.

5. R. Katz, S. C. Sharma, and M. Homayoonfar, Nucl. Instr. and Meth. 100 (1972) 13.

6. R. Katz and S. C. Sharma, Nucl. Instr. and Meth. 110 (1973) 93.

7. R. Katz and F. E. Pinkerton, Nucl. Instr. and Meth. 130 (1975) 105.

8. R. Katz and S. C. Sharma, Phys. Med. Biol. 20 (1975) 410; R. Katz, in Biological and environmental effects of low level radiation (IAEA, Vienna, 1976) pp. 343-360.

9. $\mathrm{H}$. Dertinger and $\mathrm{H}$. Jung, Molecular radiation biology (Springer Verlag, New York, 1970).

10. K. Becker, Solid state dosimetry (CRC Press, Cleveland, Ohio 44128, 1973).

11. J. R. Cameron, N. Suntharalingam, and G. N. Kenney, Thermoluminescent dosimetry (University of Wisconsin Press, Madison, 1968).

12. Proc. Int. Conf. on Luminescence dosimetry, Stanford 1965 (ed. F. H. Attix; USAEC, CONF-650637, 1967).

13. Proc. 2nd Int. Conf. on Luminescence dosimetry, Gatlinburg, Tennessee, 1968 (eds. J. A. Auxier, K. Becker and E. M. Robinson; USAEC, CONF-680920, 1968).

14. Proc. 3rd Int. Conf. on Luminesscence dosimetry, Riso, Denmark, 1971 (ed. V. Mejdahl; Danish Atomic Energy Commission. Riso Report No. 249, 1971).
15. Proc. 4th Int. Conf. on Luminescence dosimetry, Krakow, Poland, 1974 (ed. T. Niewiadomski; Institute of Nuclear Physics, Krakow, 1974).

16. W. Binder, S. Disterhoft, and J. R. Cameron, ref. 13, pp. 43-53.

17. E. Tochilin, N. Goldstein, J. T. Lyman, and W. G. Miller, U.S. Naval Radiological Defence Laboratory, San Francisco 94135, Report No. NRDL-TR-68-129 (1968). Also ref. 13, pp. 424-437.

18. J. R. Cameron, D. W. Zimmerman, and R. W. Bland, ref. 12, pp.47-56.

19. C. L. Wingate, E. Tochilin, and N. Goldstein, ref. 12, pp 421-434.

20. M. J. Aitken, M. S. Tite, and S. J. Fleming, ref. 12, pp 490-501.

21. D. W. Zimmerman, Radiat. Eff. 14 (1972) 81.

22. B. Jahnert, Health Phys. 23 (1972) 112.

23. I. Jaek, F. Savikhin, and H. Kaambre, ref. 15, pp. 565-580.

24. E. Piesch and B. Burgkhardt, ref. 15, pp. 1124-1140.

25. J. W. Patrick, L. D. Stephens, R. H. Thomas, and L. S. Kelly, Health Phys. 30 (1976) 295; also Health Phys. 28 (1975) 615.

26. E. L. Geiger and A. E. Doles, Separate identification of neutron and gamma exposures of lithium fluoride (TLD-100) used for personnel dosimetry, in Proc. 9th Midyear Topical Symp. of the Health Physics Society, Denver, Colorado (February 1976). Compiled by the Proc. Committee of the Rocky Mountain Chapter of the Health Physics Society, P. L. Carson, W. R. Hendee, and D. C. Hunt. 